\title{
Mitochondrial Impairment Mechanism in D-galactose-induced Senescence in Experimental Fibroblast Cell Model
}

\author{
Wei YE ${ }^{1,2}$, Jian-Ming CAO ${ }^{1}$, Xiao-Jun TANG ${ }^{1,2}$, Chu LIU ${ }^{1,2}$, \\ Dong-Jie HAO ${ }^{1}$, Jing $\mathrm{JIN}^{1}$, Jian-Xin $\mathrm{LU}^{1,2, *}$ \\ ${ }^{1}$ School of Laboratory Medicine and Life Science, Wenzhou Medical University, \\ Wenzhou, Zhejiang, China \\ ${ }^{2}$ Key Laboratory of Laboratory Medicine, Ministry of Education of China, School of \\ Laboratory Medicine and Life Science, Zhejiang Provincial Key Laboratory of Medical \\ Genetics, Wenzhou Medical University, Wenzhou, Zhejiang 325035, China
}

${ }^{*}$ Corresponding author

Keywords: D-galactose, Mitochondria, Oxidative Stress, Oxidative Phosphorylation.

\begin{abstract}
To establish an effective model for cellular senescence, human embryo lung fibroblast cells (MRC-5) were cultured in D-galactose (D-Gal) medium respectively, as control, same concentration of D-glucose (D-Glu) was used. Decrease in cell proliferation, increase in senescence associated $\beta$-galactosidase activity, up-expression of p21 protein, and cell cycle arrest at S-phage were observed in D-Gal-treated cells. Meanwhile, D-Gal-treated cells showed the significant increased ROS and MDA level and decreased SOD activity. Furthermore, mitochondrial impairment and decrease in efficiency of oxidative phosphorylation (OXPHOS) was induced by D-Gal as evidenced by the decreased transmembrane potential, reduction of ATP production and changes of respiration function. Additionally, the significant decrease of mitochondrial quantity, mitochondrial DNA (mtDNA) copy number and increased mtDNA damage were detected in D-Gal-treated cells. Our data demonstrate that D-Gal induces mitochondrial oxidative impairment associated with increased generation of ROS, ultimately inhibiting the ATP synthesis which contributes to premature cellular senescence.
\end{abstract}

\section{Introduction}

Aging is known to be characterized by a decline in cellular function and a decrease in the cellular metabolism ability. Mitochondria play a key role in maintaining cellular metabolism and increasing evidence has pointed out age-associated mitochondrial dysfunction $[1,2]$.

The understanding of the pathogenesis of the mitochondrial dysfunction during the aging process or at the early stage of these neurodegenerative diseases would shed light on the pathogenesis of cognitive deterioration, paving the way for better therapeutic strategies. Hence it is necessary to establish an aging animal model or cell model. At present, D-galactose (D-Gal) inducing aging mice have been acceptable, while this is not well established in the senescent cell model.

Galactose is a kind of monosaccharide as a natural energy substrate in daily food, which is transported into cell via glucose transporter and converted into glucose through series of enzymatic reactions known as Leloir pathway. Experimentally, D-Gal has been widely used for modeling mice aging by inducing age-related cognitive dysfunction and neurodegeneration [3, 4]. D-Gal application on cells is often to assess mitochondrial toxicity induced by drugs or some toxicants [5, 6] or evaluate 
mitochondrial oxidative capacity [7]. However, it is seldom used to establish cell senescence model. Cells cultured in medium with low concentrations $(5 \mathrm{mM})$ of galactose show a significantly increased oxidative phosphorylation (OXPHOS) and respiration rate compared to the cells grown in glucose-containing medium $[5,7,8]$. Although these studies show that reactive oxygen species (ROS) can be generated during the course of D-Gal metabolism, it is still unclear on the underlying mechanisms of D-Gal-induced mice aging with cognitive deterioration and neurodegenerative diseases.

In present study, we focus on establishing an effective model of cell senescence using human embryo fibroblast and demonstrating the role and mechanisms of mitochondrial dysfunction in aging. In our study, firstly modeling cell senescence in vitro was performed by culturing cells in $5 \mathrm{mM}, 25 \mathrm{mM}$ and $55 \mathrm{mM}$ (low, medium and high dose) of D-Gal and D-glucose (D-Glu), then premature cellular senescence in D-Gal and D-Glu medium were evaluated. By assessing mitochondrial respiratory function, energy metabolism, cellular redox state, mitochondrial quantity and mitochondrial DNA (mtDNA) damage, the underlying mitochondrial impairment mechanism in D-Gal induced cell senescence was investigating. We hope this study could provide informative data in the research for aging and age-associated diseases, especially the therapy.

\section{Materials and Methods}

\section{Cell Culture}

Human embryo lung fibroblast cells (MRC-5) were routinely cultivated at $37^{\circ} \mathrm{C}$ in humidified $95 \%$ air 5\% CO2 in high glucose (25 mM) DMEM medium, supplemented with $10 \%$ fetal bovine serum (FBS, Gibco, USA), $100 \mathrm{U} / \mathrm{ml}$ penicillin solution and $0.1 \mathrm{mg} / \mathrm{ml}$ streptomycin solution. For D-Galactose treatment, $2.5 \times 105 / \mathrm{ml}$ suspension cells were incubated in 6-well plates for $24 \mathrm{~h}$ when cells stick to the wells. Then the medium was replaced by $5 \mathrm{mM}, 25 \mathrm{mM}$ and $55 \mathrm{mM}$ of D-Gal separately, and cells were cultured for an additional 72h. As a control, part of MRC-5 cells grew in same concentration of D-Glucose medium.

\section{Measurement of Cell Proliferation by CCK8 Assay}

CCK8 assay is based on a reaction in which the formed NAD(P)H reduces WST-8 into formazan and the yellow intensity is proportionate to the $\mathrm{NAD}(\mathrm{P})+\mathrm{NAD}(\mathrm{P}) \mathrm{H}$ in the cells. In brief, MRC-5 cells were incubated in 96-well plates as $2 \times 103$ cells/well and the cell proliferation was detected once every $24 \mathrm{~h}$ by CCK 8 assay. The cells were washed by $1 \times$ PBS and then incubated with $10 \mu \mathrm{l}$ of CCK 8 solution in $100 \mu 1$ medium at $37^{\circ} \mathrm{C}$ for $1 \mathrm{~h}$. The absorbance of formazan which developed as a result of WST-8 reduction was measured at $450 \mathrm{~nm}$.

\section{$\beta$-galactosidase Staining}

Senescence-associated $\beta$-galactosidase (SA- $\beta$-Gal) activity was detected by using SA- $\beta$-Gal staining kit. Due to different proliferation of cell in the two kinds of medium, initial cell number in D-Gal and D-Glu medium was adjusted. The cells were cultivated for $72 \mathrm{~h}$ when cell fusion degrees were $90 \%$. Then medium was removed and the cells were washed twice with $1 \times \mathrm{PBS}$. After fixing with $1 \mathrm{ml}$ fixative solution for $15 \mathrm{~min}$ at room temperature, cells were washed with $1 \times \mathrm{PBS}$ for three times, and then stained with 
staining solution at $37^{\circ} \mathrm{C}$ overnight. The development of blue color in the cells was observed under an optical microscope (Nikon, Japan).

\section{Analysis of Cell Cycle by FCM}

$2 \times 105$ cells $/ \mathrm{ml}$ MRC-5 cells were collected and suspended in $200 \mu 1$ PBS. The suspended cells then were added into the $4 \mathrm{ml} 75 \%$ ethanol for fixation overnight at $4^{\circ} \mathrm{C}$. After centrifugation, the supernatant was discarded and the cells were re-suspended in PBS with Rnase and incubation at $37^{\circ} \mathrm{C}$ for $45 \mathrm{~min}$. Before detection on FCM, the cells were stained with PI for $30 \mathrm{~min}$ on ice.

\section{Determination of SOD Activity, MDA Content and ROS Level}

Total superoxide dismutase (SOD) activity was determined with the assay kit (Biovision, USA) using DU800 spectrophotometer (Beckman, USA) at $450 \mathrm{~nm}$. Malonaldehyde (MDA) content was determined using the commercial assay kits following the manufacturer's protocol. MDA assay was performed based on the amount of formed thiobarbituric acid reactive substances (TBARS)-MDA complex, which was detected at $535 \mathrm{~nm}$.

In measurement of ROS, the treated cells were collected and then stained by fluorescence probe DCFH-DA $(10 \mu \mathrm{mol} / \mathrm{L}, 1: 800$ diluted with serum free medium) and incubated for $20 \mathrm{~min}$ in the dark at $37^{\circ} \mathrm{C}$. Before detection, the cells were washed with serum free medium three times. The analysis was performed on a flow cytometer (FCM, BD FACS Aria I).

\section{Mitochondrial Morphology by Transmission Electron Microscopy (TEM)}

Cells were spun and washed with normal saline. After pre-fixation at $4{ }^{\circ} \mathrm{C}$ with $2.5 \%$ glutaraldehyde for $2 \mathrm{~h}$, the cells were washed with $0.1 \mathrm{M}$ phosphate buffer three times, each time for $45 \mathrm{~min}$. After fixed with $1 \%$ osmic acid for $1 \mathrm{~h}$ and washed with $0.1 \mathrm{M}$ phosphate buffer three times, the cells were incubated in $1 \%$ uranyl acetate for $2 \mathrm{~h}$, and then dehydrated in acetone concentration-gradient $(70 \%, 80 \%, 90 \%, 100 \%)$ followed by embedding in Epon 812 and polymerized at $65^{\circ} \mathrm{C}$ for $48 \mathrm{~h}$. Ultra-thin sections were obtained using an ultra-microtome (PT-XL PowerTome Ultramicrotomes, RMC, USA) and picked up onto 200 mesh copper grids. The sections were double-stained with uranyl acetate and lead citrate and imaged under a Hitachi H-7500 Transmission Electron Microscope with a Gatan BioScan 1K CCD camera at 70kV (Gatan, USA).

\section{Measurement of Mitochondrial Membrane Potential and Mitochondrial Contents by FCM}

Mitochondrial membrane potential $(\Delta \Psi \mathrm{m})$ was detected with JC-1 mitochondrial membrane potential detection kit (Sigma, USA) following the manufacturer's instructions. Briefly, $1 \times 106$ cells were incubated in $0.5 \mathrm{ml}$ of working solution with JC- 1 dye at $37^{\circ} \mathrm{C}$ for $30 \mathrm{~min}$ in the dark. The excessive dye was removed by washing with $1 \times \mathrm{JC}-1$ staining buffer. The fluorescence value of the cells suspended in $1 \mathrm{ml}$ PBS was detected in FCM.

For mitochondrial contents analysis, the cells were collected and stained with Mito-tracker (1:1000 diluted with serum free medium) in the dark at $37^{\circ} \mathrm{C}$ for $20 \mathrm{~min}$. Before detection, the cells were washed with serum free medium for three times. 


\section{Measurement of ATP by High Performance Liquid Chromatography (HPLC)}

Cells were collected in $0.1 \mathrm{~mol} / \mathrm{L}$ perchloric acid solution and homogenized on ice. The homogenate was centrifuged at $4^{\circ} \mathrm{C}$ at $14000 \mathrm{rpm}$ for $10 \mathrm{~min}$. The supernatant was acquired for measurement of ATP by HPLC analysis.

A stock solution was prepared by dissolving $5 \mathrm{mg}$ of ATP (Sigma, USA) in $15 \mathrm{ml}$ ultrapure water and stored at $4{ }^{\circ} \mathrm{C}$. For HPLC analysis, $20 \mu \mathrm{l}$ of standards or sample was injected into Agilent 1100 Series HPLC system with a C18 reverse phase hydrophilic column $\left(4.6^{*} 250 \mathrm{~mm}, 5 \mu \mathrm{m}, \mathrm{pH} 2 \sim 8\right.$, Ecosil). Detections were operated using $50 \mathrm{mM}$ monopotassium phosphate and $10 \%$ methyl alcohol as mobile phase with a flow rate of $0.5 \mathrm{ml} / \mathrm{min}$ and wavelength of $254 \mathrm{~nm}$. The retention time of each ingredient was confirmed by that of the standards. The standard curve was established by plotting the values of a series of standards diluted from the stock solutions. Concentration of ATP was quantified according to the standard curve.

\section{Analysis of Mitochondrial Oxidative Respiratory Function}

Cellular respiration was measured on Seahorse XF96 analyzer (Seahorse Bioscience). We performed mitochondrial bioenergetics assays based on previous published protocols.

$1.5 \times 103 /$ well of the treated cells were incubated on the analysis plates overnight to attach and obtain a monolayer of cells. Mitochondrial respiration test was performed by sequential additions of $1 \mu \mathrm{M}$ oligomycin, $1 \mu \mathrm{M}$ FCCP and $1 \mu \mathrm{M}$ rotenone. The following mitochondrial parameters were determined: basal mitochondrial respiration, maximal respiratory capacity, spare respiratory capacity, ATP turnover-driven respiration, proton leak and non-mitochondrial respiration. The results were expressed as OCR (Oxygen Consumption Rate) in $\mathrm{pmol}(\mathrm{O} 2) / \mathrm{min} /(\mu \mathrm{g}$ protein)

\section{Quantification of MtDNA Copy Number}

mtDNA copy number was quantified using fluorescence-based quantitative real-time PCR. In brief, PCR was performed by amplification of the human mitochondrial ND1(mtND1) gene and nuclear $\beta$-globin (HBB) gene, mtND1 or HBB copy number was interpolated from the linearity of the dose-dependent standard curves by constructed plasmids with mtND1 or HBB. Calculation of mtDNA copy number was $2 \times \mathrm{mtND} 1 / \mathrm{HBB}$.

The primers and probes for mtND1 and HBB were shown as follows. mtND1 forward primer: 5'CCCTAAAACCCGCCACATCT3', reverse primer: 5' GAGCGATGGTGAGAGCTAAGGT3', 69bp, probe: 5' (FAM) CCATCACCCTCTACATCACCGCCC (TAMRA) 3'; HBB forward primer: 5' AAAGGTGCCCTTGAGGTTGTC 3', reverse primer: 5' TGAAGGCTCATGGCAAGAAA $\quad 3^{\prime}, \quad 76 \mathrm{bp}, \quad$ probe: $5^{\prime} \quad$ (FAM) CTCTCTCCCATCCCTTCTCCCCA(TAMRA) 3'.

A total PCR reaction volume of $20 \mu 1$, containing $10 \mu 12 \times$ HS qPCR Master Mix, 0.5 $\mu \mathrm{l}$ of $10 \mu \mathrm{M}$ of each primer, $0.5 \mu \mathrm{l}$ of the $10 \mu \mathrm{M}$ probe, $6.5 \mu \mathrm{l} \mathrm{H} 2 \mathrm{O}$ and $2 \mu \mathrm{l}$ DNA template. Real time PCR was performed on an ABI StepOne Plus ${ }^{\mathrm{TM}}$ Real-time quantitative PCR System. The PCR reaction conditions were as follows: 1 cycle at $95^{\circ} \mathrm{C}$ for $2 \mathrm{~min}$, and 40 cycles at $95^{\circ} \mathrm{C}$ for $10 \mathrm{sec}, 60^{\circ} \mathrm{C}$ for $1 \mathrm{~min}$.

\section{Oxidized MtDNA Analysis by QPCR}

Oxidized mtDNA analysis was completed by qPCR as previous report [9]. Briefly, the degree of oxidized mtDNA is reflected by the abundance of $8-\mathrm{OH}-\mathrm{dG}$ formation in 
mtDNA, and it is presented as $\triangle \mathrm{Ct}$ value, which is the $\mathrm{Ct}$ difference value with and without treatment of hOGG1, an 8-oxoguanine DNA glycosylase (New England Biolab, USA). The primers amplifying mtDNA D-loop show as follows: forward primer 5' ATATCCCGCACAAGAGTGCT 3', reverse primer 5' GGGAACGTGTGGGCTATTTA 3'. PCR reaction volume and conditions were similar as mtDNA copy number detection except for the probe.

\section{Results}

\section{Senescence Characteristics Presented in D-Galactose-treated Cells}

When glucose medium was replaced by D-Gal, significant changes in cell morphology were observed (Fig.1 A). The cells presented hypertrophy, the long axis shortened, and showed rhombus or triangle. The nucleus gets bigger and nucleolus became obvious. Meanwhile, the cell proliferation of the D-Gal-treated cell was slower than controls as results show (Fig.1 B). After $\beta$-galactosidase staining, a significant increase of blue-stained MRC-5 cells was seen in the D-Gal-treated group compared to the controls (Fig.1 C). The p21 expression of the cells cultured in D-Gal medium compared with which in D-Gal show obvious higher, especially in 25mM and 55mM D-Gal (Fig.1 D). In the cell cycle analysis, the significant cell cycle arrest in S-phage was observed in D-Gal-treated cells, especially in 25mM D-Gal-treated cells (Fig.1 E, F).

\section{D-Galactose Induces Mitochondrial Structural Alteration and Dysfunction}

The pathological alteration of mitochondria originated from the mitochondrial morphology and function abnormalities. Subcellular structure of D-gal-treated cells and controls were examined by TEM analysis. Obvious alteration of mitochondrial morphology was observed in D-gal-treated cells, which mitochondria were swollen and appeared vacuolus. The double membrane was disintegrated and the cristae were unclear and even disappeared (Supl. Fig.1).

To test the energy metabolic changes after D-Gal treatment, ATP contents were measured by HPLC. Significant reduction was observed in ATP contents in the D-Gal-treated cells (Fig.2A). To investigate whether D-Gal induces the impairment of mitochondrial function, $\Delta \Psi \mathrm{m}$ was measured firstly. As results shown in Fig.2B, D-Gal treatment significantly decreased $\Delta \Psi \mathrm{m}$. CCK 8 reduction capacity also is a commonly used parameter for mitochondrial dysfunction, in D-gal-treated cells, the level of CCK8 reduction decreased significantly compared with the controls after $72 \mathrm{~h}$ ( $<<0.001$,Fig.1B).

In further experiments, Seahorse XF96 analyzer was used to detect the mitochondrial respiration. Compare with the same concentration of D-Glu, spare respiratory capacity, maximal respiration, and ATP-turnover-driven respiration in D-Gal-treated cells significantly increased (Fig.2 C-F). In D-Gal-treated cells, spare respiratory capacity shows dose-dependent increase, however, maximal respiration, ATP-turnover-driven respiration and non-mitochondrial respiration were similar in $25 \mathrm{mM}$ and $55 \mathrm{mM}$ of D-Gal.

\section{D-Galactose Increases Oxidative Stress}

Two oxidative stress parameters (MDA and SOD) and ROS level were analyzed. Compared with the controls, SOD activity was decreased and MDA content was increased significantly in D-Gal-treated cells (Fig.3 A, B). ROS production was 
assessed using the fluorescence probe DCFH-DA. As seen in Fig. $3 \mathrm{C}, 25 \mathrm{mM}$ and $55 \mathrm{mM}$ of D-Gal-treated cells produce more ROS compared with the controls.

\section{D-Galactose Decreases Mitochondrial Quantity and MtDNA Quality}

Additionally, compared to the controls, both of mitochondrial quantity and mtDNA copy number decreased in D-Gal treatment (Fig.4 A, B), and more oxidized mtDNA (ox-mtDNA) in D-Gal-treated cells was detected (Fig.3 C). In these results including the oxidative stress data, though there is no strict consistency in results of the groups of $5 \mathrm{mM}$ and $55 \mathrm{mM}$, the $25 \mathrm{mM}$ groups always keep the same results.

\section{Discussion}

Mitochondria are not only responsible for predominant sites of cellular bioenergetics, but also the producers and primary targets of ROS. Mitochondrial electron transport chain (ETC) is the cellular bioenergetics mechanism used for energy synthesis from oxidative respiration, as well as the major source of ROS, which is produced by electron leakage from ETC complexes in mitochondria during normal oxidative respiration [10]. However, the overproduction of ROS is responsible for the mitochondrial dysfunction, which is presumably due to the changes in the activity of key components of the respiratory chain or in mitochondrial bioenergetics $[11,12]$. Therefore mitochondrial dysfunction is characterized by decreased OXPHOS and increased generation of ROS, which were reported to be associated with aging or neurodegenerative diseases, such as Alzheimer's and Parkinson's diseases [1,2].

The present study shows that galactose increases ROS generation, induces of mitochondrial impairments, ultimately inhibiting production of ATP, which may lead to premature cellular senescence.

For establishment and evaluation of premature cell senescence by D-Gal, MRC- 5 cells in high glucose medium originally were transferred into the medium with $5 \mathrm{mM}$, 25mM, 55mM D-Gal separately. It was observed that the D-Gal-treated cells presented with decreased growth rate and cell proliferation, which is consistent with a previous study [7], as well as the distinct changes in cell morphology (Fig.1 A, B). Except for cell proliferation and cellular morphology, $\beta$-galactosidase activity is also a specific biomarker for senescence widely used because of the simplicity of the assay method [13]. These enzymes function optimally at $\mathrm{pH} 4$, its activity is also detectable at pH6 in senescent cells. Results indicate that D-Gal induces more premature cells senescence than glucose (Fig. 1 C).

Cell cycle arrest is attributed to cell growth suppression. Our observation that D-gal-induced S phase arrest in cell cycle and p21 up-regulation (Fig.1 D-F) was in agreement with the previous reports that the cancer cells were treated with the chemicals [14]. p21 is an inhibitor of CDKs and its up-expression usually induces cell cycle arrest at the G1 phase by inhibiting CDKs activity [15], however it also directly inhibit DNA synthesis by binding to proliferating cell nuclear antigen (PCNA)[16], which induce $S$ phage arrest. Additionally, it was proved that the expression of p21 can be induced by DNA-damaging agents via the ATM-chk2-p21 pathway [17]. In this case, D-Gal may lead to DNA damage and activate the pathway, which induces p21 expression.

For better understanding of the cellular response to the changes in environment, the substantial cellular events should be determined. The alteration of mitochondria induced by D-Gal was evident from the analysis of mitochondrial structure and function. After D-Gal treatment, the impairment of mitochondrial structural integrity 
was revealed by the destruction of membrane and cristae structure. The loss of functional integrity of the mitochondria was proved by inactivation of mitochondrial dehydrogenases and decrease of $\Delta \Psi \mathrm{m}$ (Fig.1 B, Fig.2 B). Redox environment measured by CCK8 assay was significantly lower in D-Gal-treated cells, which indicates a decrease in dehydrogenase activity and reduced $\mathrm{NAD}(\mathrm{P}) \mathrm{H}$ levels in D-Gal-treated cells compared to the controls. A similar result was also found by others in the cells grown in $10 \mathrm{mM}$ galactose compared to $25 \mathrm{mM}$ glucose $[7,18] . \Delta \Psi \mathrm{m}$ is vital for electron transport and ATP production. The data indicates that D-Gal-induced cell senescence was associated with the collapse of $\Delta \Psi \mathrm{m}$, and the collapse was demonstrated to be related to the level of oxidation (Supl. Fig.2).

Mitochondria are responsible for the final common pathway of the oxidative metabolism and ATP production. Many types of cells grown under high glucose conditions show diminished oxidative metabolism. It is known as the Crabtree effect, which means the cells generate ATP mostly via the glycolysis pathway and inhibit OXPHOS. Glycolytic metabolism of glucose to pyruvate in cytoplasm yields 2 net ATP, whereas glycolytic metabolism of galactose yields no net ATP. Thus, galactose may force cells to rely on OXPHOS pathway to generate sufficient ATP for cell growth [7, 18]. As shown in previous studies, when a high glucose condition was replaced by a galactose medium, cells might be forced to change their ATP generation pathway from glycolysis predominantly to OXPHOS exclusively in order to maintain ATP levels. Many types of cells (e.g., cancer cells, primary fibroblasts, muscle cells) show significantly increased oxygen consumption rate, expression level of respiration chain components, and mitochondrial enzymatic activities when cells were grown in galactose medium instead of glucose medium $[5,7,8,18]$. Therefore galactose medium often was used for analyzing the effect of mitochondrial toxin in cancer cells $[5,19]$ or examining mitochondrial function of skin fibroblasts from patients with mitochondrial diseases [20, 21]. In our study, the increased mitochondrial oxidative respiration (Fig.2 C-F) in the D-Gal-treated cells indicated the increased OXPHOS level. Nevertheless it also shows the decline in ATP content, which was considered in cellular oxidative damage in the process of OXPHOS.

Mitochondria are the main source of ROS and the sensitive targets of ROS as well. Increased ROS can cause oxidative damage of cellular macromolecules, including mitochondrial proteins, lipids and DNA etc. For evaluating the cellular oxidative stress, MDA content and SOD activity were determined. Higher MDA levels and lower SOD activity in D-Gal-treated cells indicate D-Gal induces the increase in oxidative stress (Fig.3 A, B). This was further confirmed by ROS determination by DCFH-DA staining on FCM (Fig.3 C). The results reveal that D-Gal induces generation of ROS and leads to mitochondrial oxidative stress, which may be the main cause of mitochondrial pathology. Higher concentration $(25 \mathrm{mM}$ and $55 \mathrm{mM})$ of D-Gal used in our study rather than $5 \mathrm{mM}$ or $10 \mathrm{mM}$ used by others might be one of the reasons of declined ATP production. Although low concentration of D-Gal develops OXPHOS initially, more D-Gal may induce overproduction of ROS and consequently oxidative damage in mitochondria, far more than its improvement in OXPHOS. On the other hand, lower mitochondrial quantity and mtDNA copy number in D-Gal-treated cells also can be explained the decline of ATP production.

In addition, D-Gal induced the significant higher level of oxidized mtDNA with 8-OH-dG, which further proved the oxidative damage in the D-Gal-treated mitochondria, and also contribute to the premature cell senescence. 

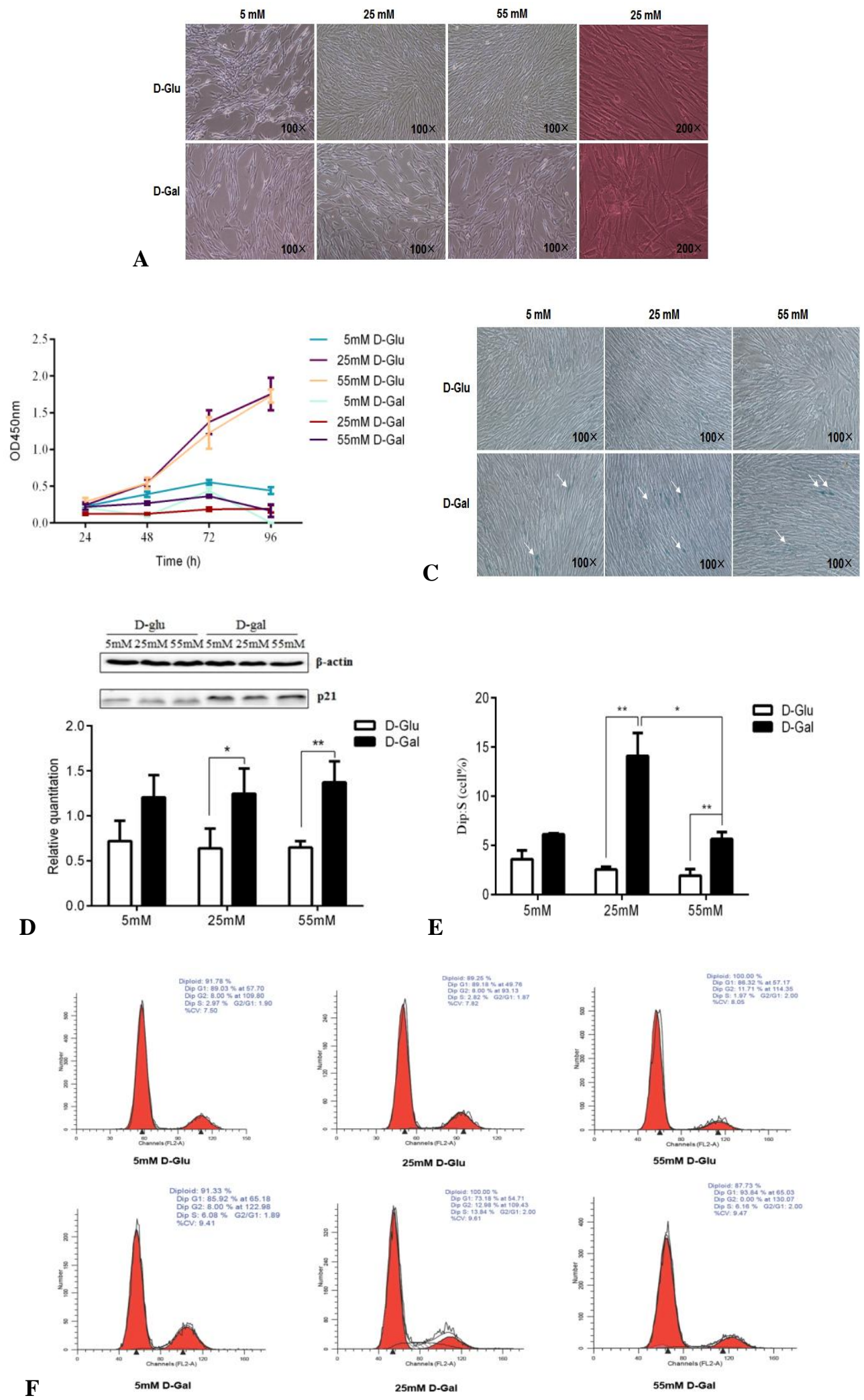

Figure 1. D-gal-treated cells presented senescent characteristics. A, B, C: Cell morphology, cell proliferation and senescence-associated $\beta$-galactosidase activity in D-Glu- and D-Gal-treated MRC-5 cells, 100x, 200×; D: p21 protein expression levels between different groups by western-blot analysis; E, F: Cell cycle analysis by FCM; *, $p<0.05$, ** $p<0.01$, D-Gal groups versus D-Glu groups, $\mathrm{n}=6$ 
A

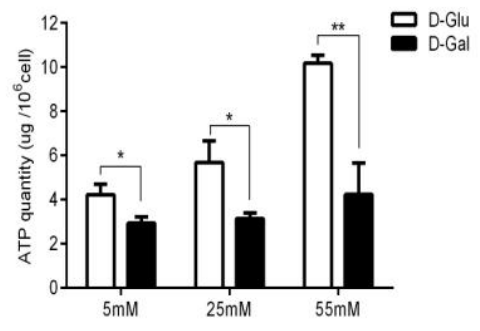

B

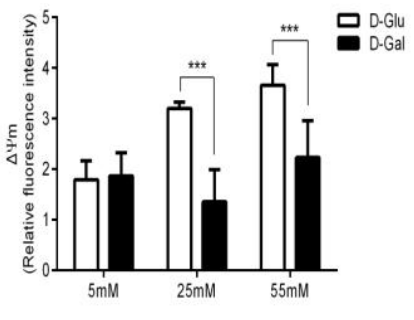

C

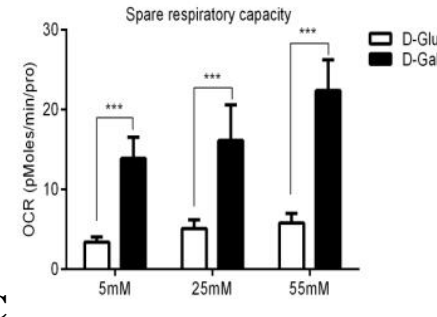

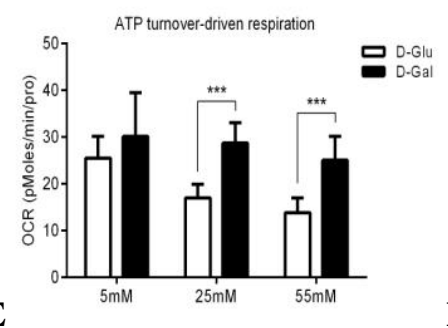

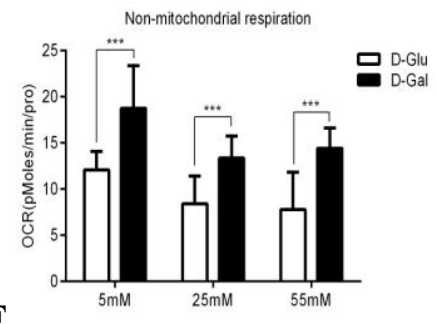

D

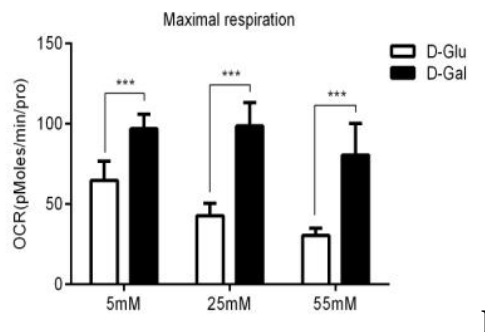

$\mathbf{E}$

F

Figure 2. D-gal induces impairment of mitochondrial function. A: ATP quantity analysis by HPLC; B: Mitochondrial membrane potential $(\Delta \Psi \mathrm{m})$ measured by JC-1 staining on FCM; C F : Mitochondrial respiration measured by Seahorse in D-Glu- and D-gal-treated cells. Data are presented as the mean \pm S.E, ${ }^{*}, p<0.05, * *, p<0.01, * * *, p<0.001, \mathrm{D}-\mathrm{Gal}$ groups versus D-Glu groups, $n=6$.
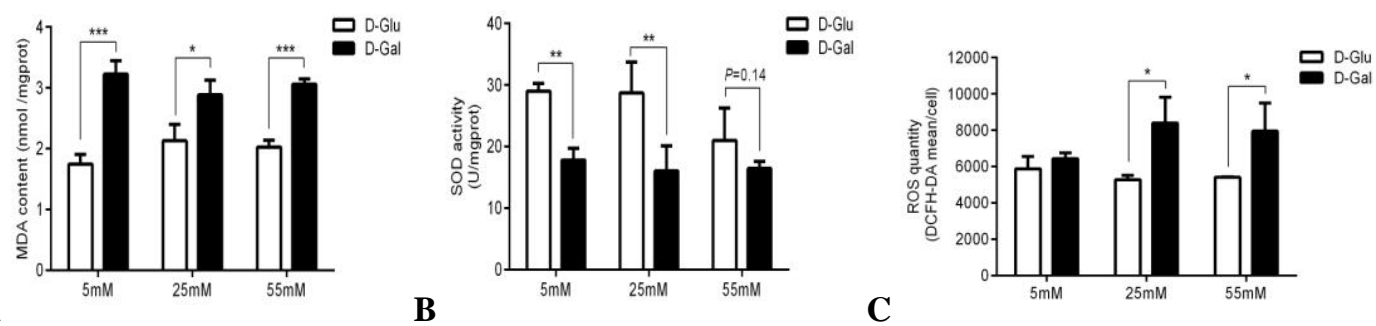

Figure 3. D-gal induces increased oxidative stress. A, B: MDA content and SOD activity in D-Glu- and D-Gal-treated cells; C: ROS detection with DFHA staining by FCM. Data are presented as the mean \pm S.E, *, $p<0.05, * *, p<0.01, * * *, p<0.001$, D-Gal groups versus D-Glu groups, $n=6$.

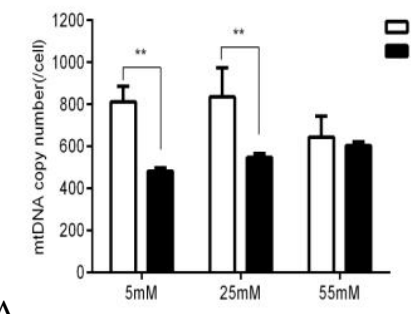

A

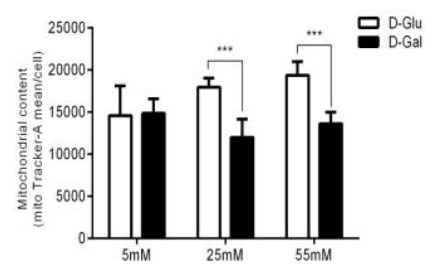

B

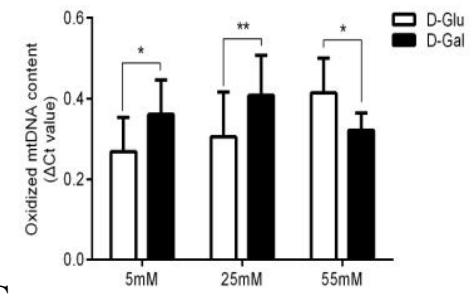

$\mathrm{C}$

Figure 4. D-Galactose decreases mitochondrial content and increases oxidized mtDNA. A: mtDNA copy number was detected by absolute quantification of qPCR; B: mitochondrial content was measured by FCM;C: Oxidized mtDNA detected by qPCR; *, $p<0.05, * *, p<0.01$, ***, $p<0.001$, D-Gal groups versus D-Glu groups, $n=6$.

\section{Conclusion}

In summary, the results imply that medium and high concentration $(25 \mathrm{mM}$ and $55 \mathrm{mM})$ of D-Gal in $72 \mathrm{~h}$ can cause structural and functional impairment of mitochondria by ROS over-generation and lead to premature cell senescence in vitro in MRC-5 
fibroblast cells. The D-Gal-related decrease in energy synthesis could be involved in the sharply increased OXPHOS, which due to the supplement of D-Gal and consequently ROS over-generation in ETC induces acute oxidative stress. In this study, we provide a feasible and effective cell senescence model induced by D-Gal, which could be potentially used in elucidating mitochondrial metabolism and function during aging in vivo, and hopefully further applied on the evaluation of the potential therapeutic targets to treat age-associated diseases.

\section{Supporting Information}

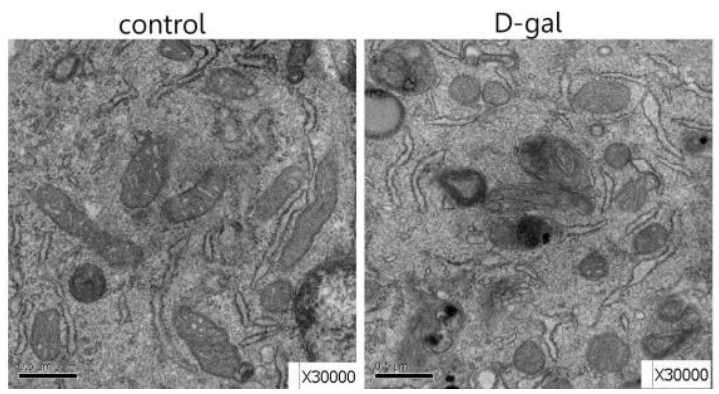

Supl. Figure 1. Morphological change of mitochondria induced by D-Gal.

Mitochondria became swollen with disintegrated double membrane and unclear cristea.

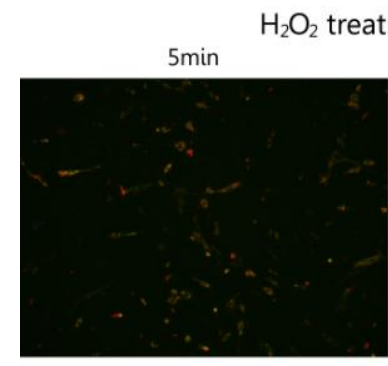

$15 \mathrm{~min}$

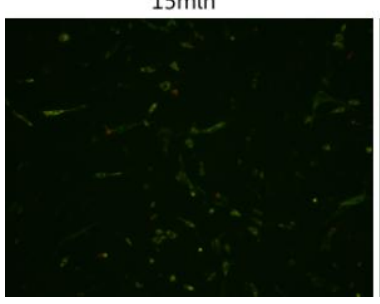

Supl. Figure 2. Collapse of $\Delta \Psi \mathrm{m}$ under $10 \mu \mathrm{M} \mathrm{H}_{2} \mathrm{O}_{2}$ in MRC5 cells

Mitochondrial membrane potential $(\Delta \Psi \mathrm{m})$ measured by JC-1 staining on Laser Scanning Confocal Microscope (LSCM). JC-1 monomer is green fluorescence (530 $\mathrm{nm})$, J-aggregate is red fluorescence (590nm). Mitochondrial membrane depolarization at a decreased ratio of red to green fluorescence indicates the decreased $\Delta \Psi \mathrm{m}$.

\section{Acknowledgement}

This work is supported by Zhejiang Provincial Natural Science Foundation (Y2100582, LY13H070006). 


\section{References}

[1] Carvalho C, Correia SC, Santos RX, et al.: Role of mitochondrial-mediated signaling pathways in Alzheimer disease and hypoxia. J Bioenerg Biomembr. 41(5): 433-40.

[2] Winklhofer KF, Haass C: Mitochondrial dysfunction in Parkinson's disease. Biochim Biophys Acta. 1802(1): 29-44.

[3] Parameshwaran K, Irwin MH, Steliou K, Pinkert CA: D-galactose effectiveness in modeling aging and therapeutic antioxidant treatment in mice. Rejuvenation Res. 13(6): 729-35.

[4] Wei H, Li L, Song Q, Ai H, Chu J, Li W: Behavioural study of the D-galactose induced aging model in C57BL/6J mice. Behav Brain Res. 157(2): 245-51.

[5] Marroquin LD, Hynes J, Dykens JA, Jamieson JD, Will Y: Circumventing the Crabtree effect: replacing media glucose with galactose increases susceptibility of HepG2 cells to mitochondrial toxicants. Toxicol Sci. 97(2): 539-47.

[6] Tsiper MV, Sturgis J, Avramova LV, et al.: Differential mitochondrial toxicity screening and multi-parametric data analysis. PLoS One. 7(10): e45226.

[7] Rossignol R, Gilkerson R, Aggeler R, Yamagata K, Remington SJ, Capaldi RA: Energy substrate modulates mitochondrial structure and oxidative capacity in cancer cells. Cancer Res. 64(3): 985-93.

[8] Shulga N, Wilson-Smith R, Pastorino JG: Sirtuin-3 deacetylation of cyclophilin D induces dissociation of hexokinase II from the mitochondria. J Cell Sci. 123(Pt 6): 894-902.

[9] Lin CS, Wang LS, Tsai CM, Wei YH: Low copy number and low oxidative damage of mitochondrial DNA are associated with tumor progression in lung cancer tissues after neoadjuvant chemotherapy. Interact Cardiovasc Thorac Surg. 7(6): 954-8.

[10] Cadenas E, Davies KJ: Mitochondrial free radical generation, oxidative stress, and aging. Free Radic Biol Med. 29(3-4): 222-30.

[11] Sastre J, Pallardo FV, Vina J: The role of mitochondrial oxidative stress in aging. Free Radic Biol Med. 35(1): 1-8.

[12] Lenaz G, Bovina C, D'Aurelio M, et al.: Role of mitochondria in oxidative stress and aging. Ann N Y Acad Sci. 959: 199-213.

[13] Maier AB, Westendorp RG, D VANH: Beta-galactosidase activity as a biomarker of replicative senescence during the course of human fibroblast cultures. Ann N Y Acad Sci. 1100: 323-32.

[14] Zhu H, Zhang L, Wu S, et al.: Induction of S-phase arrest and p21 overexpression by a small molecule 2[[3-(2,3-dichlorophenoxy)propyl] amino]ethanol in correlation with activation of ERK. Oncogene. 23(29): 4984-92.

[15] Gulappa T, Reddy RS, Suman S, Nyakeriga AM, Damodaran C: Molecular interplay between cdk4 and p21 dictates G0/G1 cell cycle arrest in prostate cancer cells. Cancer Lett. 337(2): 177-83. 
[16] Luo Y, Hurwitz J, Massague J: Cell-cycle inhibition by independent CDK and PCNA binding domains in p21Cip1. Nature 1995; 375(6527): 159-61.

[17] Buscemi G, Ricci C, Zannini L, Fontanella E, Plevani P, Delia D: Bimodal regulation of p21(waf1) protein as function of DNA damage levels. Cell Cycle.13(18): 2901-12.

[18] Aguer C, Gambarotta D, Mailloux RJ, et al.: Galactose enhances oxidative metabolism and reveals mitochondrial dysfunction in human primary muscle cells. PLoS One. 6(12): e28536.

[19] Rana P, Nadanaciva S, Will Y: Mitochondrial membrane potential measurement of $\mathrm{H} 9 \mathrm{c} 2$ cells grown in high-glucose and galactose-containing media does not provide additional predictivity towards mitochondrial assessment. Toxicol In Vitro. 25(2): 580-7.

[20] Robinson BH, Petrova-Benedict R, Buncic JR, Wallace DC: Nonviability of cells with oxidative defects in galactose medium: a screening test for affected patient fibroblasts. Biochem Med Metab Biol. 48(2): 122-6.

[21] Enriquez JA, Cabezas-Herrera J, Bayona-Bafaluy MP, Attardi G: Very rare complementation between mitochondria carrying different mitochondrial DNA mutations points to intrinsic genetic autonomy of the organelles in cultured human cells. J Biol Chem. 275(15): 11207-15. 